Bull. Austral. Math. Soc.

16D80, 16D50, 16P70

VoL. 53 (1996) [249-260]

\title{
MODULES ARISING FROM SOME RELATIVE INJECTIVES
}

\author{
YIQIANG ZHOU
}

\begin{abstract}
A ring $R$ is a right $S I$-ring if every singular right $R$-module is injective, while $R$ is a right $S^{3} I$-ring if every singular semisimple right $R$-module is injective. In this paper, we investigate and characterise several analogues of the two notions to modules, with many illustrative examples included.
\end{abstract}

\section{INTRODUCTION}

Let $R$ be an associative ring with identity and $M$ a unitary right $R$-module. In this paper we study the following conditions on the module $M$ :
$\mathcal{C}_{11}$ : Every singular $R$-module is $M$-injective;
$\mathcal{C}_{12}$ : Every singular semisimple $R$-module is $M$-injective;
$\mathcal{C}_{13}: \quad M$ is a $G V$-module and $M / \operatorname{Soc}(M)$ is locally Noetherian;
$\mathcal{C}_{14}$ : Every cyclic singular $R$-module in $\sigma[M]$ is $M$-injective;
$\mathcal{C}_{21}$ : Every $M$-singular $R$-module is $M$-injective;
$\mathcal{C}_{22}$ : Every $M$-singular semisimple $R$-module is $M$-injective;
$\mathcal{C}_{23}: M$ is a $G C O$-module and $M / \operatorname{Soc}(M)$ is locally Noetherian;
$\mathcal{C}_{24}$ : Every cyclic $M$-singular $R$-module is $M$-injective.

When $M_{R}=R_{R}, \mathcal{C}_{11}=\mathcal{C}_{14}=\mathcal{C}_{24}=\mathcal{C}_{21}$ coincides with the right $S I$-rings introduced and studied by Goodearl [3], while $\mathcal{C}_{12}=\mathcal{C}_{13}=\mathcal{C}_{23}=\mathcal{C}_{22}$ is the defining condition of the right $S^{3} I$-rings due to Page-Yousif [12]. For the various characterisations of right $S I$-rings and right $S^{3} I$-rings, we refer to [3], [11] and [12]. Modules with $\mathcal{C}_{11}$ were investigated in Yousif [18], while modules satisfying $\mathcal{C}_{21}$ constitute the main subject of Huynh-Wisbauer [8]. Article [12] considered modules with $\mathcal{C}_{12}$ and Wisbauer [16] carried out a study of modules satisfying $\mathcal{C}_{2 j}(j=1,2,3)$. We note that all these existing results on modules $M$ with $\mathcal{C}_{i j}$ required some additional assumptions on $M$ such as $M$ being quasi-projective, or finitely generated, or both (for example, see [8, $1.3 ; 2.2],[12$, Corollary 1.6$],[16,3.5 ; 3.10]$ and $[18,2.4 ; 2.6])$.

\section{Received 4 May 1995}

Copyright Clearance Centre, Inc. Serial-fee code: 0004-9729/96 \$A2.00+0.00. 
One purpose of the present paper is to exhibit several characterisations of modules $M$ satisfying $\mathcal{C}_{i j}(i=1,2 ; j=1,2,3)$ without additional assumptions on $M$. These characterisations extend naturally the known characterisations of right $S I$-rings and right $S^{3} I$-rings and improve several results in [12], [16] and [18]. The other purpose of this paper is to show the differences among these conditions. $\mathcal{C}_{11}$ implies $\mathcal{C}_{21}$, but the converse does not hold by an example in [8]. $\mathcal{C}_{12}$ does not imply $\mathcal{C}_{11}$ because of the existence of a Noetherian $V$-ring which is not a right $S I$-ring (see [11, p.347]). We shall construct examples which, together with the above-mentioned examples, establish the following implication diagram with none of these arrows (except $\mathcal{C}_{14} \Leftarrow \mathcal{C}_{11}$ ) reversible:

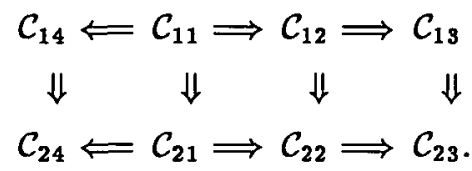

\section{DEFINITIONS AND NOTATION}

Throughout all rings $R$ are associative rings with identity and all modules are right unitary $R$-modules (unless specified otherwise). Mod- $R$ will denote the category of unitary right $R$-modules. For modules $M$ and $N, N \hookrightarrow M$ means that $N$ is embeddable in $M$, while $N \leqslant_{e} M$ means that $N$ is essential in $M$. We write $N \stackrel{e}{\rightarrow} M$ to indicate that $N$ is essentially embeddable in $M$. We denote by $Z(M)$ the singular submodule of $M$. The module $M$ is called a Goldie torsion module if $Z(M) \leqslant_{e} M$. We let $\operatorname{Soc}(M)$ be the socle of $M$, and use $\operatorname{Soc}_{n_{1}}(M)$ to indicate the sum of all non-singular simple submodules of $M$. Following [17], for any module $M$, we denote by $\sigma[M]$ the full subcategory of Mod- $R$, whose objects are the submodules of $M$ generated modules. The $M$-injective hull, $E_{M}(N)$, of $N$ is defined to be the trace of $M$ in the injective hull $E(N)$ of $N$, that is $E_{M}(N)=\sum\{f(M): f \in \operatorname{Hom}(M, E(N))\}$. Following [16], a module $N$ is called $M$-singular if $N \cong L / K$ for an $L \in \sigma[M]$ and $K \leqslant_{e} L$. Note that every $M$-singular module belongs to $\sigma[M]$. The class of all $M$ singular modules is closed under submodules, factor modules, and direct sums. Hence any module $N \in \sigma[M]$ contains a largest $M$-singular submodule, which is denoted by $Z_{M}(N)$ (see [16]). A module $N$ is said to be non $M$-singular if $Z_{M}(N)=0$. We denoted by $\operatorname{Soc}_{n_{2}}(N)$ the sum of the non $M$-singular simple submodules of $N$.

We let $\mathcal{G}(M)$ be the singular torsion theory in $\sigma[M]$, that is, $\mathcal{G}(M)$ is the smallest torsion class in $\sigma[M]$ which contains all $M$-singular modules (see [15]). $\mathcal{G}(M)$ is closed under $M$-injective hulls by $[15,2.4(3)]$, and hence $\mathcal{G}(M)=\left\{N \in \sigma[M]: Z_{M}(N) \leqslant_{e} N\right\}$.

A module is said to be locally Noetherian if every finitely generated submodule is Noetherian. A module $M$ is called a $V$-module (or $G V$-module, or $G C O$-module, respectively) if every simple (or singular simple, or $M$-singular simple, respectively) 
module is $M$-injective (see [5] and [14] and [16]). It is easy to show that submodules, factor modules, and direct sums of locally Noetherian (or $V_{-}$, or $G V_{-}$, or $G C O_{-}$, respectively) modules are locally Noetherian (or $V_{-}$, or $G V_{-}$, or $G C O_{-}$, respectively) modules. Clearly, every $G V$-module is a $G C O$-module, but the converse is not true (see [16]).

\section{Conditions $\mathcal{C}_{12}, \mathcal{C}_{13}, \mathcal{C}_{22}$ and $\mathcal{C}_{23}$}

Lemma 1 follows from [9, Theorem 1.7] and the fact that $M$ is locally Noetherian if and only if every direct sum of $M$-injective modules is $M$-injective [9, Theorem 1.11 ] if and only if every direct sum of $M$-injective hulls of simple modules is $M$-injective [19, Corollary 2.7]. It also follows from [4, Theorem 3.8] by taking the module class $\mathcal{X}$ to be Mod- $R$.

LEMMA 1. The following are equivalent for a module $M$ :

(a) $M$ is a locally Noetherian $V$-module;

(b) Every semisimple module is $M$-injective;

(c) Every countably generated semisimple module is $M$-injective.

LEMma 2. For a GCO-module $M, M$ is Noetherian if and only if every factor module of $M$ has finitely generated socle.

Proof: By an argument used in the proof of [7, Lemma 1], one can show that every GCO-module contains a maximal submodule. Since every subquotient of a $G C O$-module is $G C O$, we have the equivalence by Shock [13, Theorem 3.8].

Extending a result of right $S^{3} I$-rings in $[12$, Corollary 2.16], Wisbauer [16, 3.5] characterised quasi-projective modules with $\mathcal{C}_{23}$. Note that we have $Z_{M}(M) \cap$ $\operatorname{Soc}(M)=0$ for any quasi-projective $G C O_{\text {module }} M$ by $[16,2.3]$. Therefore the following result, characterising modules with $\mathcal{C}_{23}$, is an improvement of $[16,3.5]$.

Proposition 3. The following are equivalent for a $G C O$-module (in particular, for a $G V$-module) $M$;

(a) $M / \operatorname{Soc}(M)$ is a locally Noetherian module;

(b) Every direct sum of $M$-injective modules is $M / S o c(M)$-injective;

(c) Every direct sum of $M$-singular $M$-injective modules is $M / \operatorname{Soc}(M)$ injective;

(d) Every cyclic (or finitely generated) $M$-singular module has finitely generaged socle;

(e) $M / N$ is locally Noetherian for every essential submodule $N$ of $M$.

Moreover if $Z_{M}(M) \cap S o c(M)=0$, then (a)-(e) are also equivalent to

(f) Every $M$-singular semisimple module is $M$-injective. 
Proof: Clearly (a) $\Rightarrow(b) \Rightarrow$ (c).

(c) $\Rightarrow$ (a). Let $X=\oplus E_{\bar{M}}\left(X_{i}\right)$, where $\bar{M}=M / \operatorname{Soc}(M)$ and $X_{i} \in \sigma[M / \operatorname{Soc}(M)]$ are simple modules. By $[16,1.3]$, each $X_{i}$ is $M$-singular. Then $X_{i}$ is $M$-singular $M$ injective since $M$ is $G C O$. It follows that $X_{i}$ is $\bar{M}$-injective and hence $E_{\bar{M}}\left(X_{i}\right)=X_{i}$. Then $X=\oplus X_{i}$ is a direct sum of $M$-singular $M$-injective (simple) modules $X_{i}$. By (c), $X$ is $M / \operatorname{Soc}(M)$-injective. Therefore, by [19, Corollary 2.7], $M / \operatorname{Soc}(M)$ is locally Noetherian.

(a) $\Rightarrow$ (d). Every finitely generated $M$-singular module $N$ is in $\sigma[M / \operatorname{Soc}(M)]$ by $[16,1.3]$, and hence is Noetherian by (a). So $N$ has finitely generated socle.

(d) $\Rightarrow$ (e). Let $X \leqslant \mathrm{e} M$ and $N / X$ be a cyclic submodule of $M / X$. Then every factor module of $N / X$ is cyclic $M$-singular, and so has finitely generated socle by (d). So $N / X$ is Noetherian by Lemma 2 .

(a) $\Leftrightarrow$ (e) follows from the fact that for a module $P, P / \operatorname{Soc}(P)$ is Noetherian if and only if $P / X$ is Noetherian for all $X \leqslant_{e} P$ (see [12, Corollary 2.9]).

A similar argument in the proof of "(c) $\Rightarrow(a)$ " shows (f) $\Rightarrow$ (e). And a similar argument in the proof of "(b) $\Rightarrow$ (c)" of $[18,3.5]$ shows $(a)+(b) \Rightarrow(f)$.

For a module $M$ with $Z_{M}(M) \cap S o c(M)=0$, we have $\mathcal{C}_{22} \Leftrightarrow \mathcal{C}_{23}$ by Proposition 3. In general for modules with $\mathcal{C}_{22}$ we have the following result.

Proposition 4. The following are equivalent for a module $M$ :

(a) Every $M$-singular semisimple module is $M$-injective;

(b) $M / S o c_{n_{2}}(M)$ is locally Noetherian V-module;

(c) $M$ is a $G C O$-module and $M / \operatorname{Soc}_{n_{2}}(M)$ is locally Noetherian;

(d) $M$ is a GCO-module and every direct sum of $M$-singular $M$-injective modules is $M$-injective;

(e) $M$ is a GCO-module and every cyclic (or finitely generated) module in $\mathcal{G}(M)$ has finitely generated socle.

ProOF: (a) $\Rightarrow$ (b). In view of Lemma 1 , we need show that every semisimple module is $M / S_{o c_{n_{2}}}(M)$-injective. Since every $M$-singular semisimple module is $M$ injective by (a) and hence $M / S o c_{n_{2}}(M)$-injective, it suffices to show that every non $M$-singular semisimple module is $M / \operatorname{Soc}_{n_{3}}(M)$-injective.

Let $X$ be a non $M$-singular semisimple module, and $A / S_{o n_{2}}(M)$ an essential submodule of $M / \operatorname{Soc}_{n_{2}}(M)$, and $f: A / S_{o n_{n_{2}}}(M) \rightarrow X$ an $R$-homomorphism. Let $\operatorname{Ker}(f)=B / S_{o c_{n_{2}}}(M)$. We claim that $B \leqslant_{e} A$. If not, then $B \cap Y=0$ for some nonzero submodule $Y$ of $A$. Therefore, we have $Y \cong(Y+B) / B \hookrightarrow A / B \hookrightarrow X$. Then $Y$ is non $M$-singular semisimple, and thus $Y \subseteq S_{o c_{n_{2}}}(M) \subseteq B$, a contradiction. Therefore, $B \leqslant_{e} A$. Then, from $A / B \hookrightarrow X, X$ is not non $M$-singular unless $A=$ $B$. Therefore, $f=0$, and so $f$ can trivially be extended to a homomorphism from 
$M / \operatorname{Soc}_{n_{2}}(M)$ to $X$.

(b) $\Rightarrow$ (c). Let $X$ be an $M$-singular simple module, $N$ an essential submodule of $M$, and $f: N \rightarrow X$ an $R$-homomorphism. Note that $S_{o c_{n_{2}}}(M) \subseteq \operatorname{Ker}(f)$. Therefore, $f$ induces an $R$-homomorphism $\bar{f}: N / \operatorname{Soc}_{n_{3}}(M) \rightarrow X$ by $\bar{f}(\bar{a})=f(a)$ for all $\bar{a} \in$ $N / \operatorname{Soc}_{n_{2}}(M)$. Since $M / \operatorname{Soc}_{n_{2}}(M)$ is a $V$-module, there is an $R$-homomorphism $g$ from $M / S_{o n_{n_{2}}}(M)$ to $X$ that extends $\bar{f}$. Let $\pi: M \rightarrow M / \operatorname{Soc}_{n_{2}}(M)$ be the canonical $R$-homomorphism. Then $g \circ \pi: M \rightarrow X$ is a homomorphism that extends $f$. Therefore, $X$ is $M$-injective.

The implication (c) $\Rightarrow$ (d) can be proved by [9, Theorem 1.11] and an argument similar to that in the proof above. The proof of $(d) \Rightarrow(a)$ is obvious.

(e) $\Rightarrow$ (b). First we note that every simple module in $\sigma\left[M / S o c_{n_{2}}(M)\right]$ is $M$ singular. To see this, let $E$ be a simple module in $\sigma\left[M / S_{\text {oc }_{n_{2}}}(M)\right]$. By an argument similar to that in $[16$, p.4238], we have an essential submodule $N$ of $M$ with an epimorphism

$$
\phi: N \longrightarrow N / S \operatorname{coc}_{n_{2}}(M) \longrightarrow E .
$$

If $E$ is not $M$-singular, then the maximal submodule $\operatorname{Ker}(\phi)$ of $N$ is not essential in $N$, and thus $\operatorname{Ker}(\phi)$ is a direct summand of $N$. Then $N=\operatorname{Ker}(\phi) \oplus E^{\prime}$ with $E^{\prime} \cong E$. Therefore, $E^{\prime}$ is non $M$-singular and so $E^{\prime} \subseteq \operatorname{Soc}_{n_{2}}(M)$, implying $E^{\prime} \subseteq \operatorname{Ker}(\phi)$ and $\operatorname{Ker}(\phi)=N$. This is a contradiction.

Now let $X$ be a cyclic module in $\sigma\left[M / \operatorname{Soc}_{n_{2}}(M)\right]$. There is a submodule $Y$ of $X$ such that $\operatorname{Soc}(X) \cap Z_{M}(N) \stackrel{e}{\hookrightarrow} X / Y$. Then $X / Y$ is in $\mathcal{G}(M)$, and thus $\operatorname{Soc}(X) \cap Z_{M}(X)$ is finitely generated by (i). Therefore, by the note above, $\operatorname{Soc}(X)=\operatorname{Soc}(X) \cap Z_{M}(X)$ is finitely generated. Now the implication follows from Lemma 2.

(b) $\Rightarrow$ (e). For a finitely generated module $X$ in $\mathcal{G}(M)$, we have $X \in \sigma\left[M / S_{o c} n_{n_{2}}(M)\right]$. In fact we have $X \stackrel{e}{\leftrightarrow} M^{(I)} / A$ for some index set $I$ and a submodule $A$ of $M^{(I)}$. If $\operatorname{Soc}_{n_{2}}\left(M^{(I)}\right) \nsubseteq A$, then $\left[\operatorname{Soc}_{n_{2}}\left(M^{(I)}\right)+A\right] / A$ has a non $M$-singular simple submodule which is embeddable in $X$, a contradiction. So $\operatorname{Soc}_{n_{2}}\left(M^{(I)}\right) \subseteq A$ and hence $X \in \sigma\left[M / \operatorname{Soc}_{n_{2}}(M)\right]$. Then (b) implies that $X$ is Noetherian and hence has finitely generated socle.

Remark. It is true that Proposition 4 still holds when " $M$-singular $M$-injective modules" in statement (d) is replaced by " $M$-injective modules in $\mathcal{G}(M)$ ". But this is not the case if "cyclic (or finitely generated) modules in $\mathcal{G}(M)$ " in statement (e) is replaced by "cyclic (or finitely generated) $M$-modules". The fact is that $M$ satisfies $\mathcal{C}_{23}$ if and only if $M$ is $G C O$ and every cyclic (or finitely generated) $M$-singular module has finitely generated socle (by Proposition 2). We shall give example of a module satisfying $\mathcal{C}_{23}$ but not $\mathcal{C}_{22}$ later.

A proof analogous to that of Proposition 4 yields the next result, which improves 
[12, Corollary 1.5; Corollary 1.9].

Proposition 5. The following are equivalent for a module $M$ :

(a) Every singular semisimple module is $M$-injective;

(b) $M / \operatorname{Soc}_{n_{1}}(M)$ is a locally Noetherian $V$-module;

(c) $M$ is a $G V$-module and $M / S_{o n_{n_{1}}}(M)$ is locally Noetherian;

(d) $M$ is a $G V$-module and every direct sum of singular $M$-injective modules is $M$-injective;

(e) $M$ is a $G V$-module and every cyclic (or finitely generated) Goldie torsion module in $\sigma[M]$ has finitely generated socle.

Remark. Proposition 5 still holds when "singular $M$-injective modules" in statement (d) is replaced by "Goldie torsion $M$-injective modules". But this is not the case if "cyclic (or finitely generated) Goldie torsion modules" in statement (e) is replaced by "cyclic (or finitely generated) singular modules". In fact, we have the following implications: " $M$ satisfies $\mathcal{C}_{12}$ " $\Rightarrow$ " $M$ is $G V$ and every cyclic (or finitely generated) singular module in $\sigma[M]$ has finitely generated socle" $\Rightarrow$ " $M$ satisfies $\mathcal{C}_{13}$ ". In Section 3 we shall construct a module $M$ which satisfies $\mathcal{C}_{13}$ but some cyclic singular module in $\sigma[M]$ has infinitely generated socle. We have been unable to find an example of a $G V$-module $M$ without $\mathcal{C}_{13}$ but for which every cyclic singular module in $\sigma[M]$ has finitely generated socle.

We end this section by giving an example.

EXAMPLE 6: There exists a module $T_{R}$ such that

(a) $T_{R}$ is not a $G V$-module;

(b) Every $T_{R}$-singular module is $T_{R}$-injective.

Let ${ }_{K} P$ be an infinite dimensional vector space over a field $K$ and let $T$ be the subring of End $\left.{ }_{K} P\right)$ generated by the socle of End $\left({ }_{K} P\right)$ and the scalar transformations. Then $T$ is a (two-sided) $S I$-ring by [2, p.131]. But $T$ is not a right $V$-ring (see [1, Example 25, p.234]). Let $R$ be the ring of upper triangular $2 \times 2$ matrices over $T$. The map

$$
R=\left(\begin{array}{ll}
T & T \\
0 & T
\end{array}\right) \longrightarrow T,\left(\begin{array}{ll}
a & b \\
0 & c
\end{array}\right) \mapsto a
$$

is a surjective ring homomorphism whose kernel is an essential right ideal of $R$. Under this ring homomorphism, every right $T$-module can be regarded as a right $R$-module such that, for any module $M_{T}, M_{R}$ is singular. Since $T$ is not a right $V$-ring, some simple module $M_{T}$ is not injective as $T$-module. Then the singular simple module $M_{R}$ is not $T_{R}$-injective. So $T_{R}$ is not a $G V$-module. But for any $T_{R}$-singular module $N_{R}$, $N$ can be regarded as a singular module over $T$. So $N_{T}$ is injective since $T$ is an $S I$-ring. Therefore, $N_{R}$ is $T_{R}$-injective. 
This example implies the following:

(1) There exists a $G C O$-module which is not a $G V$-module (see [16]);

(2) There exists a module with $\mathcal{C}_{23}$ but not $\mathcal{C}_{13}$;

(3) There exists a module with $\mathcal{C}_{22}$ but not $\mathcal{C}_{12}$;

(4) There exists a module with $\mathcal{C}_{21}$ but not $\mathcal{C}_{11}$ (see [8]);

(5) There exists a module with $\mathcal{C}_{24}$ but not $\mathcal{C}_{14}$.

\section{Conditions $\mathcal{C}_{11}, \mathcal{C}_{14}, \mathcal{C}_{21}$ and $\mathcal{C}_{24}$}

Rings for which every singular module is injective, called $S I$-rings, were introduced and studied in [3]. In generalising the concept to modules, two situations arise: $S I$ modules in the sense of [18], that is, modules with $\mathcal{C}_{11}$, and $S I$-module in the sense of [8], that is, modules with $\mathcal{C}_{21} \cdot \mathcal{C}_{11}$ implies $\mathcal{C}_{21}$, but the converse is not true (see [8]). For more detail on the study of the two notions, we refer to [18] and [8]. We now characterise $S I$-modules in either sense.

Proposition 7. The following are equivalent for a module $M$ :

(a) Every $M$-singular module is $M$-injective;

(b) Every factor module in $\mathcal{G}(M)$ of $M$ is semisimple;

(c) Every (finitely generated) module in $\mathcal{G}(M)$ is semisimple;

(d) Every (finitely generated) module in $\mathcal{G}(M)$ is $M$-injective;

(e) Every (finitely generated) module in $\mathcal{G}(M)$ is quasi-continuous;

(f) Every cyclic module in $\mathcal{G}(M)$ is $M$-injective;

(g) Every $M$-singular semisimple module is $M$-injective and $\operatorname{Soc}(P) \neq 0$ for every factor module $P$ in $\mathcal{G}(M)$ of $M$.

Proof: Note that $\mathcal{G}(M)$ is closed under submodules, direct sums, $M$-injective hulls, and factor modules. Therefore, applying [19, Theorem 3.5] to $\mathcal{G}(M)$, we have the equivalences $(\mathrm{c}) \Leftrightarrow(\mathrm{d}) \Leftrightarrow(\mathrm{e}) \Leftrightarrow(\mathrm{f})$. The implication (c) $+(\mathrm{d}) \Rightarrow(\mathrm{g})$ is obvious.

The remaining implications are easy to show.

EXAMPLE 8: Let $R=Z /(4)$. Then $R_{R}$ is not a right $S I$-ring since $Z\left(R_{R}\right)=2 R$ is not injective. But (1) every singular factor module of $R_{R}$ is semisimple; and hence (2) every singular $R$-module is semisimple; and hence (3) every singular $R$-module is quasi-continuous.

Osofsky [10] showed that $R$ is semisimple if and only if every cyclic module is injective. Extending this to semisimple modules, we have that $M$ is semisimple if and only if every cyclic module in $\sigma[M]$ is $M$-injective (see $[6, \mathrm{p} .127]$ or $[19$, Corollary $3.6]$ ). In [11], it was shown that $R$ is a right $S I$-ring if and only if every cyclic singular module is injective. Therefore, it is natural for one to ask the following questions: Does $\mathcal{C}_{14}$ imply $\mathcal{C}_{11}$ ? Does $\mathcal{C}_{24}$ imply $\mathcal{C}_{21}$ ? 
We shall give an example in Section 3 which shows that $\mathcal{C}_{24}$ does not imply $\mathcal{C}_{21}$ in general. But $\mathcal{C}_{21} \Leftrightarrow \mathcal{C}_{24}$ if $Z_{M}(M)=0$ as the following shows.

Corollary 9. The following are quivalent for a module $M$ with $Z_{M}(M)=0$ :

(a) Every $M$-singular module is $M$-injective;

(b) $M / N$ is semisimple for every $N \leqslant_{e} M$;

(c) Every (finitely generated) $M$-singular module is semisimple;

(d) Every (finitely generated) $M$-singular module is $M$-injective;

(e) Every (finitely generated) $M$-singular module is quasi-continuous;

(f) Every cyclic $M$-singular module is $M$-injective;

(g) $M$ is a $G C O$-module, $M / \operatorname{Soc}(M)$ is locally Noetherian, and $\operatorname{Soc}(M / N) \neq 0$ for every $N \leqslant_{e} M$.

Proof: Note that if $Z_{M}(M)=0$, then every module in $\mathcal{G}(M)$ is $M$-singular. In fact, let $X$ be in $\mathcal{G}(M)$. Then we can write $X \stackrel{e}{\hookrightarrow} M^{(I)} / A$ for some index set $I$ and a submodule $A$ of $M^{(I)}$. If $A$ is not essential in $M^{(I)}$, then $A \cap B=0$ for some $0 \neq B \subseteq M^{(I)}$. This implies that $Z_{M}(B) \neq 0$ since $Z_{M}(X) \leqslant_{e} X$. Therefore, $Z_{M}(M) \neq 0$, a contradiction. So $A \leqslant_{e} M^{(I)}$ and hence $X$ is $M$-singular. The proof follows from this and Proposition 3.

Analogous arguments yield the following results.

Proposition 10. The following are equivalent for a module $M$ :

(a) Every singular module is $M$-injective;

(b) Every Goldie torsion factor module of $M$ is semisimple;

(c) Every (finitely generated) Goldie torsion module in $\sigma[M]$ is semisimple;

(d) Every (finitely generated) Goldie torsion module in $\sigma[M]$ is $M$-injective;

(e) Every (finitely generated) Goldie torsion module in $\sigma[M]$ is quasi-continuous;

(f) Every cyclic Goldie torsion module in $\sigma[M]$ is $M$-injective;

(g) $M$ is an $S^{3} I$-module and $S o c(P) \neq 0$ for any Goldie torsion factor module $P$ of $M$;

(h) Every singular module in $\sigma[M]$ is $M$-injective.

CoROLlary 11. The following are equivalent for a non-singular module $M$ :

(a) Every singular module is $M$-injective;

(b) $M / N$ is semisimple for every essential submodule $N$ of $M$;

(c) Every (finitely generated) singular module in $\sigma[M]$ is semisimple;

(d) Every (finitely generated) singular module in $\sigma[M]$ is $M$-injective;

(e) Every (finitely generated) singular module in $\sigma[M]$ is quasi-continuous;

(f) Every cyclic singular module in $\sigma[M]$ is $M$-injective;

(g) $M$ is a $G V$-module, $M / \operatorname{Soc}(M)$ is locally Noetherian, and $\operatorname{Soc}(M / N) \neq$ 0 for any essential submodule $N$ of $M$. 
We have been unable to determine whether or not $\mathcal{C}_{14}$ implies $\mathcal{C}_{11}$.

It is known that every right $S^{3} I$-ring is right non-singular (see [12]). We now give an example of module $M$ with $\mathcal{C}_{11}$ but $0 \neq Z(M)=Z_{M}(M) \cong M / N$ for an essential submodule $N$ of $M$.

EXAMPLE 12: Let $R=\left(\begin{array}{cc}F & F \\ 0 & F\end{array}\right)$, where $F$ is a field. Then $\operatorname{Soc}\left(R_{R}\right)=\left(\begin{array}{cc}0 & F \\ 0 & F\end{array}\right)$ is the only proper essential right ideal of $R$. Let $M_{1}=\left(\begin{array}{cc}F & F \\ 0 & 0\end{array}\right), M_{2}=R / \operatorname{Soc}\left(R_{R}\right)$, and $M=M_{1} \oplus M_{2}$. Note that $M_{1}$ is non-singular and has a unique composition series of length 2. It follows that every singular $R$-module is $M_{1}$-injective. Obviously, every singular module is $M_{2}$-injective. Therefore, every singular module is $M$-injective. Let $N=\left(\begin{array}{ll}0 & F \\ 0 & 0\end{array}\right) \oplus M_{2}$. Since $\left(\begin{array}{ll}0 & F \\ 0 & 0\end{array}\right) \leqslant_{e} M_{1}, N$ is essential in $M$. And we have $Z(M)=M_{2} \cong M_{1} /\left(\begin{array}{ll}0 & F \\ 0 & 0\end{array}\right) \cong M / N$.

\section{EXAMPLES}

In this section, we construct the examples promised earlier.

LEMma 13. [20, Example 1] Let $Q=\prod_{i=1}^{\infty} F_{i}$, where each $F_{i}=Z_{2}$, and let $T$ be the subring of $Q$ generated by $\bigoplus_{i=1}^{\infty} F_{i}$ and $1_{Q}$. Then, for a right ideal $S$ of $T$,

(a) $\operatorname{Soc}\left(T_{T}\right)=\bigoplus_{i=1}^{\infty} F_{i}$ is the only proper essential right ideal of $T$ and $T / \operatorname{Soc}\left(T_{T}\right)$ is a two-element field;

(b) $T / S$ is $T$-injective if $S o c\left(T_{T}\right) / S$ is finitely generated;

(c) $T / S$ is $T$-injective if $S \nsubseteq S o c\left(T_{T}\right)$.

Example 14: There exists a module $M$ satisfying $\mathcal{C}_{13}$ but some cyclic singular module in $\sigma[M]$ has infinitely generated socle. The map

Let $T$ be as in Lemma 13 and $R=\left(\begin{array}{cc}T & T \\ 0 & T\end{array}\right)$ be the formal triangular matrix ring.

$$
R=\left(\begin{array}{ll}
T & T \\
0 & T
\end{array}\right) \longrightarrow T,\left(\begin{array}{ll}
a & b \\
0 & c
\end{array}\right) \longmapsto a
$$

is a surjective ring homomorphism whose kernel is $I=\left(\begin{array}{ll}0 & T \\ 0 & T\end{array}\right)$ which is an essential right ideal of $R$. Therefore, for a module $M_{T}, M_{R}$ is singular. It follows that the cyclic singular module $T_{R}$ has an infinitely generated socle. Note that $T_{R} / \operatorname{Soc}\left(T_{R}\right)$ is a finite module and hence Noetherian. Next, we show that every simple singular module in 
$\sigma\left[T_{R}\right]$ is $T_{R}$-injective. Let $X$ be such a module. Then $X_{T}$ is a simple module over $T$. Therefore, by Lemma $13, X$ is $T_{R}$-injective. Finally, because any simple module, if not in $\sigma\left[T_{R}\right]$, is trivially $T_{R}$-injective, $T_{R}$ is also a $G V$-module.

LEMma 15. Let $Q=\prod_{i}^{\infty} Q_{i}$, where each $Q_{i}=\left(\begin{array}{cc}\mathbf{Z}_{2} & \mathbf{Z}_{2} \\ 0 & \mathbf{Z}_{2}\end{array}\right)$, be the full product of the upper triangular rings over $\mathbf{Z}_{2}$, and let $T$ be the subring of $Q$ generated by $\bigoplus_{i=1}^{\infty} Q_{i}$ and $1_{Q}$. Let $T_{0}=\bigoplus_{i=1}^{\infty} P_{i}$, where $P_{i}=\left(\begin{array}{ll}0 & \mathrm{Z}_{2} \\ 0 & \mathrm{Z}_{2}\end{array}\right)$, and $R=T / T_{0}$. Then

(a) $\operatorname{Soc}\left(R_{R}\right)=\left(\bigoplus_{i} Q_{i}\right) /\left(\bigoplus_{i} P_{i}\right)\left(\cong \bigoplus_{i}\left(Q_{i} / P_{i}\right)\right)$ is the only proper essential right ideal of $R$ and $R / \operatorname{Soc}\left(R_{R}\right)$ is a two-element field;

(b) $R / I$ is $R$-injective for any right ideal $I$ of $R$ with $\operatorname{Soc}\left(R_{R}\right) / I$ finitely generated;

(c) $R / I$ is $R$-injective for any right ideal $I$ of $R$ with $I \nsubseteq \operatorname{Soc}\left(R_{R}\right)$.

Proof: Similar to the proof of [20, Example 1].

EXAMPLE 16: We now construct a module $M$ satisfying $\mathcal{C}_{23}$ and $\mathcal{C}_{24}$ but not $\mathcal{C}_{21}$. Let $T$ be as in Lemma 15 , and let $C=\bigoplus_{i=1}^{\infty} C_{i}$ and $D=\bigoplus_{i=1}^{\infty} D_{i}$, where each $C_{i}=\left(\begin{array}{cc}\mathbf{Z}_{2} & \mathbf{Z}_{2} \\ 0 & 0\end{array}\right)$ and $D_{i}=\left(\begin{array}{cc}0 & \mathbf{Z}_{2} \\ 0 & \mathbf{Z}_{2}\end{array}\right)$. Note that both $C$ and $D$ are ideals of $T$. Let $R=\left(\begin{array}{cc}T & T / D \\ 0 & T\end{array}\right)$ be the formal triangular matrix ring. The map

$$
R=\left(\begin{array}{cc}
T & T / D \\
0 & T
\end{array}\right) \longrightarrow T,\left(\begin{array}{ll}
a & b \\
0 & c
\end{array}\right) \longmapsto a
$$

is a surjective ring homomorphism whose kernel is $I=\left(\begin{array}{cc}0 & T / D \\ 0 & T\end{array}\right)$. We can check that $J=\left(\begin{array}{cc}D & 0 \\ 0 & 0\end{array}\right)$ is a right ideal of $R$ such that $J \subseteq S o c\left(R_{R}\right)$, and $K=\left(\begin{array}{cc}D & T / D \\ 0 & T\end{array}\right)$ is an essential right ideal of $R$. Therefore, for a module $N_{T}, N_{R}$ is singular if and only if $N K=0$ if and only if $N J=0$ if and only if $N D=0$. We consider the $T$ module $M=C \oplus(T / D)$. $S o c\left((T / D)_{T}\right)$ is $M$-singular (see Example 12) and not finitely generated. It follows that $M / \operatorname{Soc}_{n_{2}}(M)$ is not locally Noetherian since $T / D$ is cyclic. Next, we show that every cyclic $M$-singular module is $M$-injective. Let $X$ be such a module. Then $X K=0$, and so $X_{R}$ is a factor module of $R / K$. Since $(R / K) I=0$, $X_{T}$ is a factor module of $(R / K)_{T}$. Therefore, the isomorphism, $(T / D)_{T} \cong(R / K)_{T}$ by $\bar{x} \mapsto\left(\begin{array}{ll}x & 0 \\ 0 & 0\end{array}\right)+K$, shows that $X_{T}$ is a factor module of $(T / D)_{T}$. To show $X$ 
is $M$-injective, by [19, Corollary 1.8], it suffices to show that $X$ is both $C_{i}$-injective (for each $i$ ) and $T / D$-injective. The argument in Example 12 shows that $X$ is $C_{i-}$ injective. Lemma 15 will ensure that $X$ is $T / D$-injective if we can show that $X_{T}$ is semisimple. Since $X$ is $M$-singular, there exist an index set $J$ and submodules $L$ and $K$ of $M^{(J)}$ such that $K \leqslant_{e} L \leqslant_{e} M^{(J)}$ and $X \cong L / K$. Note that $\operatorname{Soc}\left(M^{(J)}\right) \subseteq K$ and $M^{(J)} / \operatorname{Soc}\left(M^{(J)}\right) \cong[M / \operatorname{Soc}(M)]^{(J)}$ is semisimple. It follows that $M^{(J)} / K$ is semisimple, implying that $X$ is semisimple. Therefore, $X$ is $M$-injective. Finally, since every $M$-singular simple module is cyclic, $M_{R}$ is a $G C O$-module.

Let $T$ be a right $S^{3} I$-ring but not a right $S I$-ring. Note that such a ring $T$ exists since there is a Noetherian $V$-ring which is not an $S I$-ring (see [11, p.347]). If $R$ is the upper triangular matrix ring over $T$, then, by an argument similar to that in Example 6, the module $T_{R}$ satisfies $\mathcal{C}_{22}$ but not $\mathcal{C}_{21}$.

\section{REFERENCES}

[1] F.W. Anderson and K.R. Fuller, Rings and categories of modules (Springer-Verlag, Berlin, Heidelberg, New York, 1994).

[2] G. Baccella, 'Generalized $V$-rings and non Neumann regular rings', Rend. Sem. Mat. Univ. Padova 72 (1984), 117-133.

[3] K.R. Goodearl, 'Singular torsion and the splitting properties', Mem. Amer. Math. Soc. 124 (1972).

[4] A. Harmanci and P.F. Smith, 'Relative injectivity and module classes', Comm. Algebra 20 (1992), 2471-2502.

[5] Y. Hirano, 'Regular modules and V-modules', Hiroshima Math. J. 11 (1981), 125-142.

[6] D.V. Huynh, N.V. Dung and R. Wisbauer, 'On modules with finite uniform and Krull dimension', Arch. Math. 57 (1991), 122-132.

[7] D.V. Huynh, P.F. Smith and R. Wisbauer, 'A note on $G V$-modules with Krull dimension', Glasgow Math. J. 32 (1990), 389-390.

[8] D.V. Huynh and R. Wisbauer, 'A structure theorem for $S I$-modules', Glasgow Math. J. 34 (1992), 83-89.

[8] S.H. Mohamed and B.J. Müller, Continuous modules and discrete modules (Cambridge University Press, Cambridge, 1990).

[10] B.L. Osofsky, 'Rings all of whose finitely generated modules are injective', Pacific J. Math. 14 (1964), 645-650.

[11] B.L. Osofsky and P.F. Smith, 'Cyclic modules whose quotients have complements direct summands', J. Algebra 139 (1991), 342-354.

[12] S.S. Page and M.F. Yousif, 'Relative injectivity and chain conditions', Comm. Algebra 17 (1989), 899-924.

[13] R.C. Shock, 'Dual generalizations of the Artinian and Noetherian conditions', Pacific J. Math. 54 (1974), 227-235.

[14] H. Tomonaga, 'On s-unital rings', Math. J. Okayama Univ. 18 (1976), 117-134. 
[15] R. Wisbauer, 'Localization of modules and central closure of rings', Comm. Algebra 9 (1981), 1455-1493.

[16] R. Wisbauer, 'Generalized co-semisimple modules', Comm. Algebra 8 (1990), 4235-4253.

[17] R. Wisbauer, Foundations of modules and ring theory (Gordon and Breach Science Publishers, 1991).

[18] M.F. Yousif, 'SI-modules', Math. J. Okayama Univ. 28 (1986), 133-146.

[19] Y. Zhou, 'Direct sums of $M$-injective modules and module classes', Comm. Algebra 23 (1995), 927-940.

[20] Y. Zhou, 'Notes on weakly-semisimple rings', Bull. Austral. Math. Soc. 52 (1995), 517-525.

Department of Mathematics

University of British Columbia

Vancouver BC

Canada V6T 122
Current address:

Department of Mathernatics and Statistics Memorial University of Newfoundland

St. John's NF A1C 5 S7

Canada 